\title{
Density-functional calculations of carbon diffusion in GaAs
}

\author{
C. D. Latham* \\ School of Physics, University of Exeter, Exeter, EX4 4QL, United Kingdom
}

M. Haugk

Institut für Physik, Theoretische Physik III, Technische Universität, D-09107 Chemnitz, Germany

R. Jones

School of Physics, University of Exeter, Exeter, EX4 4QL, United Kingdom

Th. Frauenheim

Theoretische Physik, Fachbereich Physik, Universität-GH Paderborn, D-33098 Paderborn, Germany

\author{
P. R. Briddon \\ Department of Physics, University of Newcastle upon Tyne, Newcastle, NE1 7RU, United Kingdom
}

(Received 12 May 1999)

\begin{abstract}
Self-consistent-charge density-functional tight-binding (SCC-DFTB) calculations have been performed to survey the potential-energy surface for a single interstitial carbon atom introduced into GaAs. The results provided a possible model for the diffusion of carbon through GaAs with an activation energy of less than $1 \mathrm{eV}$. The carbon atom moves via split-interstitial and bond-centered configurations. Subsequently, the energetics of the model reaction were refined using a fully self-consistent density-functional method, AIMPRO. These calculations were found to be in good agreement with the more approximate SCC-DFTB results. Experimental studies have also found an activation energy of $\sim 1 \mathrm{eV}$ for carbon migration in heavily doped material. [S0163-1829(99)02246-8]
\end{abstract}

\section{INTRODUCTION}

At low concentrations $\left([\mathrm{C}]<10^{19} \mathrm{~cm}^{-3}\right)$ the diffusion rate for isolated substitutional $\mathrm{C}_{\mathrm{As}}$ acceptors in $\mathrm{GaAs}$ is some two orders of magnitude less than other $p$-type dopants such as $\mathrm{Be}$ or $\mathrm{Zn} .{ }^{1}$ Under As-rich conditions, measurements of the activation energy for migration of $\mathrm{C}_{\mathrm{As}}$ cover the range of $2.8-3.1 \mathrm{eV}^{2-4}$ (The rate is lower under Ga-rich conditions.) Hence it is possible to create carbon-doped regions with very abrupt $p$ - $n$ boundaries which do not degrade over time. Unfortunately, the advantage which carbon has is lost when the concentration rises above $[\mathrm{C}] \approx 5 \times 10^{19} \mathrm{~cm}^{-3} \cdot 5$ In secondary-ion-mass spectrometry (SIMS) and microstructural studies the smearing of a carbon-doped region with initially sharp boundaries was observed. ${ }^{6}$ In these experiments a considerable quantity of carbon interstitials was found ( $\approx 25 \%$ of $[\mathrm{C}]$ ), which appeared to be very mobile even at growth temperatures, and therefore reduced the sharpness of the $p-n$ junction. $^{7}$ Moreover, it was observed that the annealing of highly $\mathrm{C}$-doped $\mathrm{GaAs}$ at temperatures between 600 and $850^{\circ} \mathrm{C}$ leads to a drastic reduction in the hole concentration $[p] .{ }^{8-10}$ This cannot be explained by interstitial diffusion, but instead the formation of compensating defects is required. Below $\sim 550-600^{\circ} \mathrm{C}$ or at low carbon concentrations, degradation was not observed: $[p]$ increased to a level nearly equal to $[\mathrm{C}] .^{8,10}$ In other words almost all $\mathrm{C}$ atoms were activated as acceptors, thus in these circumstances the concentration of interstitials or other compensating defects was negligible. The loss of holes is in fact seen across the whole spectrum of $\mathrm{Al}_{x} \mathrm{Ga}_{1-x} \mathrm{As}$ alloys for $x=0-1$. $^{11}$

The dominant defect responsible for hole loss has been identified in material grown by solid source molecular-beam epitaxy (MBE) as some form of carbon pairs. ${ }^{12}$ Ramanscattering observations supported by $a b$ initio theoretical modeling have shown that new dicarbon defects consisting of a pair of carbon atoms lying at an arsenic lattice site, $(\mathrm{C}-\mathrm{C})_{\mathrm{As}}^{+}$, or interstitial dicarbon defects, $(\mathrm{C}-\mathrm{C})_{i}^{2+}$, are formed when annealing heavily doped material. ${ }^{13-15}$ These defects are deep donors, or double donors, respectively, hence three or four holes are lost for each dicarbon complex formed (provided that all $\mathrm{C}$ atoms were active as acceptors intitially). Their formation implies the activation energy for carbon diffusion has a relatively low value in material where $[\mathrm{C}] \gtrsim 5 \times 10^{19} \mathrm{~cm}^{-3}$. In high-temperature annealing experiments on heavily carbon-doped GaAs, Fushimi and Wada have measured a value of $\approx 1 \mathrm{eV}$ for the activation energy of the hole loss process. ${ }^{16}$ The annealing was performed in two steps. The first step activated $\mathrm{C}_{\mathrm{As}}$ acceptors by eliminating hydrogen from the material, and the second resulted in the loss of the activated acceptors. At present, no details have yet emerged of the reaction mechanism for carbon migration, however, it is widely thought that it involves a "kick-out" process, where highly mobile arsenic interstitials $\left(\mathrm{As}_{i}\right)$ displace $\mathrm{C}_{\mathrm{As}}$ atoms. This was suggested by H. M. You et al. ${ }^{3}$

In strongly $p$-type material it would be expected that any $\mathrm{As}_{i}$ present exists in the triply ionized state $\mathrm{As}_{i}^{3+}$. Northrup and Zhang have calculated the formation energy in GaAs of $\mathrm{As}_{i}^{3+}$ to be $3.15 \mathrm{eV}+3 \mu_{e}+\Delta \mu / 2$, and $\Delta H=-1.05 \mathrm{eV}{ }^{17}$ Given that $-\Delta H \leqslant \Delta \mu \leqslant \Delta H$, and in strongly $p$-type material $\mu_{e} \approx 0 \mathrm{eV}$, the minimum formation energy of $\mathrm{As}_{i}^{3+}$ is $\approx 2.6 \mathrm{eV}$. The measured activation energy for the diffusion of carbon $(2.8-3.1 \mathrm{eV})$ lies $\sim 0.2-0.5 \mathrm{eV}$ above this which is consistent with the formation of $\mathrm{As}_{i}^{3+}$ being the slowest step. 
Thus the activation energy for the hole loss process measured by Fushimi and Wada is lower than the formation energy for $\mathrm{As}_{i}^{3+}$ as calculated previously. This suggests that there is a source of $\mathrm{As}_{i}$ present in Fushimi and Wada's specimens, which are released $\sim 400{ }^{\circ} \mathrm{C}$, and the $\mathrm{C}_{\mathrm{As}}$ atoms in effect represents a sink for them.

It has also been suggested by Fushimi and Wada that hydrogen may play a role, and the possibility of a vacancyassisted process is not excluded. ${ }^{18}$ This is supported by experiments they performed on $p^{+}-n$ junction structures similar to those used in heterojunction bipolar transistors (HBT's). The degredation of the electrical characteristics of diodes is correlated with the presence of hydrogen in the specimens. The hydrogen released from carbon forms extended defects such as platelets whose formation involves the creation of interstitials. ${ }^{19}$ Such interstitials would then be recombination centers in the device junction region. In the absence of hydrogen, however, dicarbon complexes can form in material which has been prepared so as to contain as little hydrogen as possible. Moreover, if hydrogen were present in significant quantities, then it would interact with the dicarbon complexes to form new defects containing hydrogen and carbon such as the $\left(\mathrm{C}_{\mathrm{As}}\right)_{2} \mathrm{H}$ aligned defect complex identified by Ying Cheng et al. in as-grown GaAs epitaxial layers. ${ }^{20}$ It might also be expected that $\mathrm{C}_{\mathrm{As}}-\mathrm{H}$ would also be present (this has an infrared active $\mathrm{C}-\mathrm{H}$ stretch mode at $\left.2635 \mathrm{~cm}^{-1}\right) .^{21,22}$ No such defects have been observed in the "hydrogen-free" material. In the case of the possibility of a vacancy-assisted process, it is difficult to see how this could result in dicarbon defects. Moreover, production of $\mathrm{C}_{\mathrm{Ga}}$ defects appears to be inevitable with this mechanism; these have never been observed. For a recent review of diffusion in GaAs and related compounds see Ref. 23.

In the following sections we will next examine the kickout process by using local-density-functional based theoretical methods to explore the potential energy surface for a single interstitial carbon atom in GaAs, and hence calculate reaction and activation energies for diffusion which may be compared with experiment.

\section{METHODS}

The potential-energy surface for interstitial atoms in GaAs is expected to be very complex with many local minima. There is also no information, experimental or theoretical, available from other sources about the migration path for a carbon atom through a GaAs crystal. These combined facts make it extremely difficult to choose relevant structures and constraints for modeling the diffusion mechanism directly with a fully $a b$ initio method. The approach adopted here therefore was to first conduct a survey of many possible ideas using an approximate but fast self-consistent-charge density-functional tight-binding (SCC-DFTB) method (to be described later). This enabled us to identify the main features along the migration path, which could then be examined in detail with a much higher degree of confidence using a more accurate but time-consuming method, AIMPRO. This will also be described in more detail later. The AIMPRO method was also able to provide the initial parametrization of the repulsive potentials $E_{\text {rep }}$ for the SCC-DFTB simulations, and enabled further refinements to be made from the subsequent detailed calculations for use in future work using the SCCDFTB method.

In order to search for reaction pathways using the SCCDFTB method, constraints were placed on the relaxation of the atomic coordinates in the conjugate-gradient energy minimization algorithm it uses. To simulate the migration of an atom between a chosen pair of fully relaxed local minima in energy, the total energy was calculated at points along the vector defining the direct trajectory connecting the two metastable states, while simultaneously the movement of selected atoms was restricted to the plane to which the vector is perpendicular at each point. The selection of atoms generally included (but not only) those which appeared to be bonded to different neigbours in each local minimum. Some experimentation is required to obtain a satisfactory result. If too many atoms are included in the selection there is a risk that the system is overconstrained which will result in an overestimate of the activation energy. On the other hand, if the system is underconstrained because too few atoms are selected, the energy plotted as a function of distance along the trajectory vector will not be smooth, and atoms make unreasonably large jumps in position at some point along the migration path. A strength of the SCC-DFTB method in this respect is that it is sufficiently fast that many different selections can be made until the best one is found.

Once a low-energy trajectory has been found (including its ends), it is then apparent how the atomic movements are coordinated, which bonds are exchanged, and so on. The AIMPRO method can then be employed to examine the process in detail and give more reliable estimates of energies. The system of constraints described above can be applied to the AIMPRO method, however, for reactions where an exchange of bonds can be identified the difference of the squares of the bond lengths of bonds pairs being exchanged is a better choice of reaction coordinate. For each pair of bonds being exchanged, the total energy as a function of quantity $r_{a}^{2}-r_{b}^{2}$, is calculated, where $r_{a}$ and $r_{b}$ are the bond lengths. Provided the correct pair or pairs of bonds have been chosen, this yields a smooth curve for one pair, or a saddlelike surface for two pairs. Although the method is completely general, it is not practical to model systems where more than two pairs of bonds are exchanged $-N$ pairs requires $O\left(x^{N}\right)$ complete energy minimizations.

The self-consistent-charge density-functional tightbinding (SCC-DFTB) method uses a basis of numerically derived $s$ and $p$ confined atomic orbitals derived within selfconsistent-field local-density approximation (SCF-LDA) calculations and all two-center integrals of the DFT Hamiltonian and overlap matrix are explicitly evaluated. Thus in contrast to empirical tight-binding (TB) schemes, interactions extending beyond the first shell of neighbors are taken into account. Charge redistribution is also taken into account through the incorporation of self-consistency by adjustment of the Mulliken charges based on a second-order expansion of the Kohn-Sham energy. This greatly improves the description of chemical bonding in systems containing more than one type of atom such as compound semiconductors, yet has little impact on the computational effort because this is dominated by the general eigenvalue problem. For further details of the SCC-DFTB method and its application, see Refs. 24-26. In this work, a 216-atom periodic supercell was 
constructed for the simulations.

In the AIMPRO method, the Kohn-Sham wave functions of the valence electrons are expanded in a basis set of $s$ and $p$ Gaussian orbitals, together with norm-conserving pseudopotentials for the atomic cores. ${ }^{27}$ The required orbital symmetries of the basis functions are provided by suitable multiplicative factors. The basis set used to obtain the results presented here consisted of four Gaussian-type orbitals in $s, \quad p_{x}, \quad p_{y}, \quad p_{z}$ variants, with different widths, centered on each gallium, arsenic, and carbon atom, with two Gaussian orbitals per hydrogen atom. The charge density was fitted to five $s$-type Gaussian functions for each gallium and arsenic atom, four for each carbon, and three for each hydrogen. The basis for the surface hydrogen was restricted to a fixed linear combination of Gaussian orbitals; full variational freedom was permitted for the other atoms. Supplementary Gaussian-type basis functions were placed at the center of every bond, except to the surface hydrogen atoms. The forces acting on each atom are given by an analytical formula derived from the total-energy expression. All calculations used a spin-averaged exchange-correlation energy functional, with a modified interpolation scheme for the Ceperley-Alder expression, which does not appear to suffer from the overbinding usually seen in LDA methods. ${ }^{28}$ Structural optimization to minimize the total energy is performed by a conjugate-gradient algorithm. For further details, see Ref. 29. These calculations used a 135-atom cluster. The "ideal" structure without the additional interstitial atom or any other point defects has $C_{3 v}$ symmetry, and a stochimetric formula of $\mathrm{Ga}_{34} \mathrm{As}_{34} \mathrm{H}_{66}$. The unequal distribution of $\mathrm{Ga}$ and As atoms along the $C_{3 v}$ pole of the cluster imposes a significant dipole moment on it. This can be nearly canceled out by substituting hydrogen atoms on the surface with "pseudohydrogen" atoms with fractional nuclear charges. Each surface hydrogen atom is taking the place of one quarter of a host gallium or arsenic atom which each would have one less or one more proton, respectively, than a group-IV element. Therefore, when a hydrogen atom is bonded to gallium, its proton's charge is multiplied by $5 / 4$, and $3 / 4$ when it is bonded to arsenic. As there are equal numbers of gallium and arsenic atoms at the surface of the cluster, there are equal numbers of the two types of pseudohydrogen atoms. This technique is, of course, applicable to nonstochimetric clusters such as those with $T_{d}$ symmetry, though the effect is generally much less. To enable comparisons, clusters with fractional and with integer charge surface protons were used in the calculations presented here.

The relative performance of the two methods to each other and their accuracy is worth considering briefly. In our experience, as a rule of thumb, the SCC-DFTB method is about a factor of 10 faster than the AIMPRO method. The relaxed structures produced by both methods are nearly always very similar, however, the relative accuracy of calculated energies should be considered to be in proportion to the time taken to compute them. Having said that, our experience is that relative energies of structures mostly agree to better than $0.1 \mathrm{eV}$. When the SCC-DFTB method apparently fails it usually produces an overbound result. Failure is more likely to occur for bonding configurations far from those for which $E_{\text {rep }}$ was parametrized.

It is difficult to give an estimate for the absolute accuracy of the calculations for a particular situation. Instead an example of a similar kind of problem-the diffusion of interstitial carbon in silicon-is illustrative of how well the AIMPRO method is able to perform. Experimental measurements of the energy barrier to migration and reorientation of $\mathrm{C}_{i}$ in $\mathrm{Si}$ lie in the range $0.73-0.87 \mathrm{eV} \cdot{ }^{30-32}$ Using the AIMPRO method, Leary et al. estimated the activation energy to be $1.10 \mathrm{eV} .{ }^{33}$ Since a smaller cluster (87 atoms) and a smaller basis was used than in the present work, it is reasonable to expect that the results given here for $\mathrm{C}_{i}$ in GaAs are more reliable than previously obtained for $\mathrm{C}_{i}$ in $\mathrm{Si}$. A less sophisticated procedure was also employed to determine the migration path, which tends to overestimate the barrier. Other theoretical calculations give values in the range $0.51-2.1 \mathrm{eV}$ depending on the method and the migration path (about which some controversy still exits). ${ }^{34-36}$

\section{RESULTS}

Heavily carbon-doped GaAs containing nearly all the carbon in the form of $\mathrm{C}_{\mathrm{As}}$ acceptors is very strongly $p$-type, therefore the Fermi level lies close to the top of the valence band, and any donors present will be ionized. A single carbon interstitial atom $\mathrm{C}_{i}$ inserted into the ideal GaAs lattice has two electrons occupying a level deep in the gap, hence in $p$-type material its normal state will have a +2 charge.

With the SCC-DFTB method it was very quickly established that the minimum-energy location for $\mathrm{C}_{i}^{2+}$ is at the center of a $\mathrm{Ga}-\mathrm{As}$ bond, i.e., a linear $(\mathrm{Ga}-\mathrm{C}-\mathrm{As})^{2+}$ configuration with $C_{3 \mathrm{v}}$ symmetry. The next lowest energy minimum, about $0.5 \mathrm{eV}$ above the bond-centered structure, was found to be a $(\mathrm{C}-\mathrm{Ga})_{\mathrm{Ga}}^{2+}$ split interstitial with an approximately [100]-aligned $\mathrm{C}-\mathrm{Ga}$ bond. In the third place, the corresponding $(\mathrm{C}-\mathrm{As})_{\mathrm{As}}^{2+}$ split interstitial was also metastable, and had a relative energy of about $1.0 \mathrm{eV}$.

When these three structures were tested with the AIMPRO method, the energy ordering was found to be different. The $(\mathrm{C}-\mathrm{Ga})_{\mathrm{Ga}}^{2+}$ split interstitial was lowest at $0.3 \mathrm{eV}$ below the linear $(\mathrm{Ga}-\mathrm{C}-\mathrm{As})^{2+}$ configuration, which was in turn $0.1 \mathrm{eV}$ below the $(\mathrm{C}-\mathrm{As})_{\mathrm{As}}^{2+}$ split interstitial. Assuming these figures are more reliable, it appears therefore that while the relative energies of the split interstitials agree to within $0.1 \mathrm{eV}$, the bond-centered structure is overbound with the SCC-DFTB method. Moreover, all the relevant bond lengths were slightly shorter. This indicates that the short-ranged repulsive contribution to the TB total energy was too weak for short bond lengths. This was partially remedied by choosing a new parametrization of the repulsive potential term based on all-electron self-consistent density functional calculations. The split intersitial structures now agreed in energy to better than $0.04 \mathrm{eV}$, but the bond-centered structure structure still remained lowest at about $0.2 \mathrm{eV}$ below the $(\mathrm{C}-\mathrm{Ga})_{\mathrm{Ga}}^{2+}$ complex. A possible explanation for this difference is that the carbon atom experiences a very different bonding configuration in the split-interstitial and bond-centered sites, and the SCC-DFTB method being only a minimal basis approach and not fully self-consistent is not very reliable in these circumstances. The slightly harder TB repulsive potential yielded longer bond lengths, thus bringing their values into very close agreement with the AIMPRO calculations. It is 
TABLE I. Calculated energies $(\mathrm{eV})$ of the $(\mathrm{C}-\mathrm{As})_{\mathrm{As}}^{2+}$ and $(\mathrm{Ga}-\mathrm{C}-\mathrm{As})^{2+}$ defects in GaAs relative to $(\mathrm{C}-\mathrm{Ga})_{\mathrm{Ga}}^{2+}$.

\begin{tabular}{lccc}
\hline \hline & AIMPRO & SCC-DFTB $^{\text {a }}$ & SCC-DFTB $^{b}$ \\
\hline$(\mathrm{C}-\mathrm{As})_{\mathrm{As}}^{2+}$ & +0.38 & +0.47 & +0.34 \\
$(\mathrm{Ga}-\mathrm{C}-\mathrm{As})^{2+}$ & +0.30 & -0.52 & -0.25
\end{tabular}

${ }^{\mathrm{a}} E_{\text {rep }}$ fitted to AIMPRO.

${ }^{\mathrm{b}} E_{\text {rep }}$ fitted to all-electron density-functional theory.

quite possible that the agreement may be improved by further optimization of the basis.

The effects of relaxation of the surface hydrogen and using neutral or pseudohydrogen were also considered at the AIMPRO level of theory. A selected set of calculations showed no significant difference in the relative energies of structures determined using fixed or free surface hydrogen atoms, therefore we opted to hold the hydrogen fixed as this takes slightly less computational effort. The important thing is to be consistent. Using pseudohydrogen on the other hand does turn out to be significant: the relative energies of the $(\mathrm{C}-\mathrm{As})_{\mathrm{As}}^{2+}$ split interstitial and the bond-centered structure relative to the $(\mathrm{C}-\mathrm{Ga})_{\mathrm{Ga}}^{2+}$ split interstitial are slightly more than double if "normal" hydrogen is used instead. Table I summarizes these results.

Having found these three structures, and noting that there are only relatively modest differences in energy between each of the split interstitials and the bond-centered site, it was now apparent how carbon might diffuse through the crystal. Starting from one of the two split interstitial structures, the path would take it to the other via the bondcentered structure (see Fig. 1). By performing many simulations at the SCC-DFTB level of theory in conjunction with the first method for constraining atoms we were able to examine this process and identify the main features of the atomic movements involved. The calculations, however, are not reliable enough to give meaningful energies in this situation where atoms are undergoing large changes in their bonding configuration far from those used in the initial fitting process.

The SCC-DFTB simulations showed that one pair of bonds is exchanged when the carbon moves from $(\mathrm{C}-\mathrm{As})_{\mathrm{As}}^{2+}$ to the bond-centered site, and second pair when it subsequently moves to $(\mathrm{C}-\mathrm{Ga})_{\mathrm{Ga}}^{2+}$. The bonds which break and form are illustrated by Fig. 2. Starting from $(\mathrm{C}-\mathrm{As})_{\mathrm{As}}^{2+}$, the $\mathrm{C}-\mathrm{Ga}$ bond labeled " $a$ ', breaks, then the $\mathrm{Ga}-\mathrm{As}$ bond " $b$ " forms. Next, the Ga-As bond " $c$ ", breaks, and finally the C-As bond ' $d$ " forms. Hence there are two reactions for which we can define coodinates as described previously in terms of the difference of the squares of the lengths of the exchanging pairs of bonds. For the first reaction, $(\mathrm{C}-\mathrm{As})_{\mathrm{As}}^{2+} \rightarrow(\mathrm{Ga}-\mathrm{C}-\mathrm{As})^{2+}$, this is, $r_{a}^{2}-r_{b}^{2}$; and in the case of the second reaction, $(\mathrm{Ga}-\mathrm{C}-\mathrm{As})^{2+}$ $\rightarrow(\mathrm{C}-\mathrm{Ga})_{\mathrm{Ga}}^{2+}$, it is $r_{c}^{2}-r_{d}^{2}$.

Figures 3 and 4 show how the energy varies as a function of the reaction coordinates. Starting from a $(\mathrm{C}-\mathrm{As})_{\mathrm{As}}^{2+}$ state, a migrating carbon atom faces a small barrier of only $0.09 \mathrm{eV}$ to reach a $\mathrm{Ga}-\mathrm{As}$ bond-center. From here it then encounters a somewhat higher but still relatively small barrier of $0.39 \mathrm{eV}$ when it moves to a $(\mathrm{C}-\mathrm{Ga})_{\mathrm{Ga}}^{2+}$ state. In the opposite direction, the activation energy is $0.71 \mathrm{eV}$, thus this is the largest barrier an interstitial carbon atom must overcome to move via this path.

Two alternative diffusion paths were also considered in addition to the above. At first glance it looks viable for a carbon atom to move through an interstitial cage from one $(\mathrm{C}-\mathrm{As})_{\mathrm{As}}^{2+}$ or $(\mathrm{C}-\mathrm{Ga})_{\mathrm{Ga}}^{2+}$ site to the nearest neighboring one of the same type. Efforts to simulate this always failed. In the case of $(\mathrm{C}-\mathrm{As})_{\mathrm{As}}^{2+}$ to $(\mathrm{C}-\mathrm{As})_{\mathrm{As}}^{2+}$, the carbon atom jumped to form a $(\mathrm{C}-\mathrm{Ga})_{\mathrm{Ga}}^{2+}$ when it was pushed into the interstitial space away from the arsenic atom. Similar jumping behavior also occurred in the case of diffusion from $(\mathrm{C}-\mathrm{Ga})_{\mathrm{Ga}}^{2+}$ to $(\mathrm{C}-\mathrm{Ga})_{\mathrm{Ga}}^{2+}$. The only path which could be found with smoothly varying energy along the chosen coordinate without discontinuous steps in position was the "bond-centered", one.

\section{DISCUSSION}

Only one candidate has emerged for the diffusion mechanism for interstitial carbon atoms in GaAs. It fits well with the idea that highly mobile interstitial arsenic atoms can displace $\mathrm{C}_{\mathrm{As}}$ shallow acceptors by forming a $(\mathrm{C}-\mathrm{As})_{\mathrm{As}}^{2+}$ split interstitial complex as the first step. This is reminiscent of the so-called "transient enhanced dopant diffusion" process
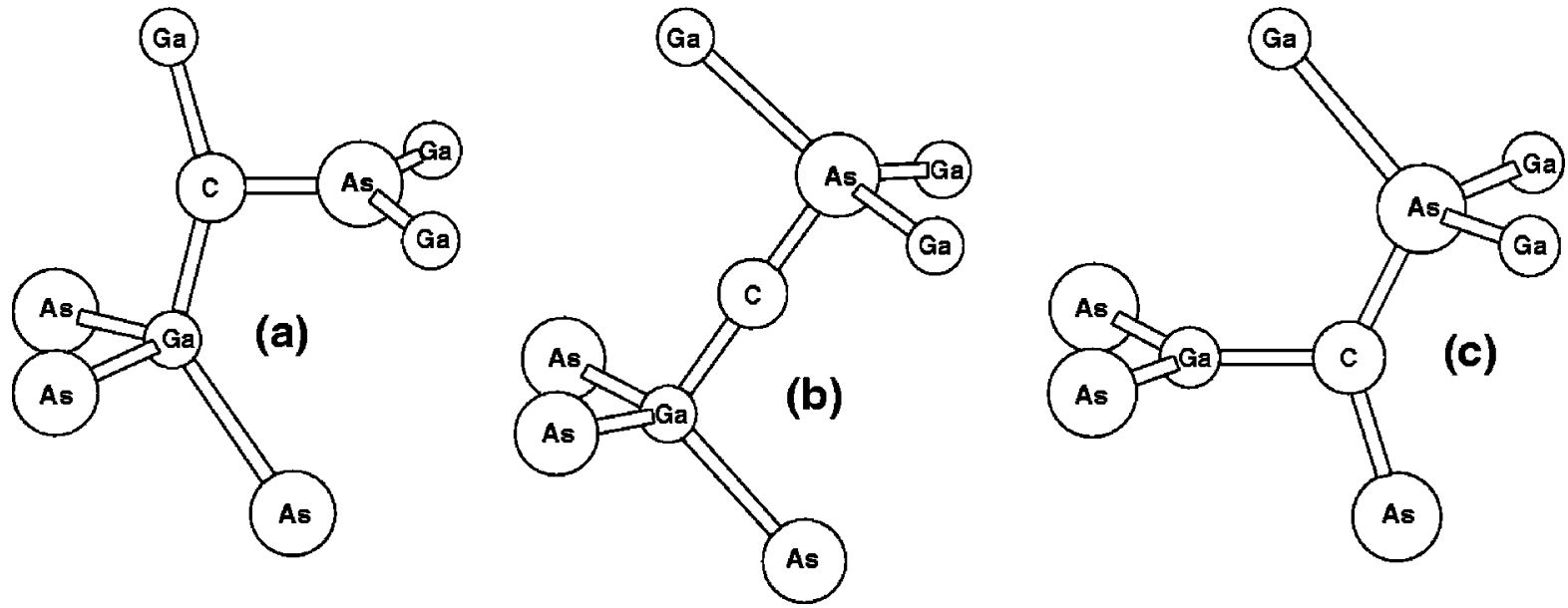

FIG. 1. (a) $(\mathrm{C}-\mathrm{As})_{\mathrm{As}}^{2+}$ split interstitial, (b) bond-centered $\mathrm{C}^{2+}$ interstitial, (c) $(\mathrm{C}-\mathrm{Ga})_{\mathrm{Ga}}^{2+}$ split interstitial. 

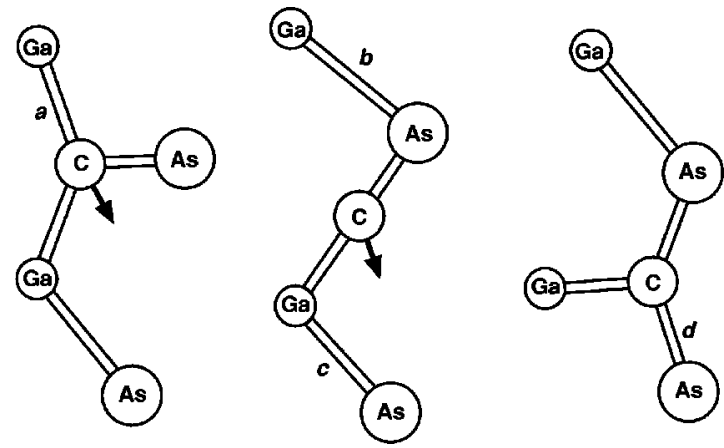

FIG. 2. Diagram showing which pairs of bonds are exchanged for the diffusion of $\mathrm{C}_{i}^{2+}$. First, " $a$ " breaks and " $b$ " forms, then " $c$ " breaks and " $d$ " forms.

in silicon. ${ }^{37}$ The enhanced diffusion of dopants is transient in that it depends on a source of interstitials which, once exhausted, stops. The source of interstitial arsenic atoms, we speculate, may be some kind of extended defect analogous to the $\{311\}$ agglomerates in $\mathrm{Si}$, or other $\mathrm{As}_{i}$ clusters. If this is the case, then the elimination of such defects would prevent this diffusion process from occuring.

Along the migration path, the most stable structure is a $(\mathrm{C}-\mathrm{Ga})_{\mathrm{Ga}}^{2+}$ split interstitial complex, thus it would be expected that the equilibrium concentration of these is higher than the bond-centered $(\mathrm{Ga}-\mathrm{C}-\mathrm{As})^{2+}$ or the $(\mathrm{C}-\mathrm{As})_{\mathrm{As}}^{2+}$ split interstitial structures. A second carbon atom encountering this defect is then conveniently placed to form the $(\mathrm{C}-\mathrm{C})_{i}$ dicarbon interstitial complex found previously. ${ }^{15}$ This is composed of one gallium and two carbon atoms sharing a $\mathrm{Ga}$ lattice site, with the carbon pair strongly bound together, but perturbed by the presence of the neighboring gallium atom. A more likely encounter, at least in the early stages of annealing, would be with a $\mathrm{C}_{\mathrm{As}}$ atom, in which case a $(\mathrm{C}-\mathrm{C})_{\mathrm{As}}$ dicarbon complex would form.

Other charge states of $\mathrm{C}_{i}$ defects are not expected to be found in heavily carbon-doped $p$-type crystals. Therefore structures such as the $\mathrm{C}(1)$ complexes found in electronirradiated GaAs where the Fermi-level lies near or above midgap are not considered. ${ }^{38-41}$

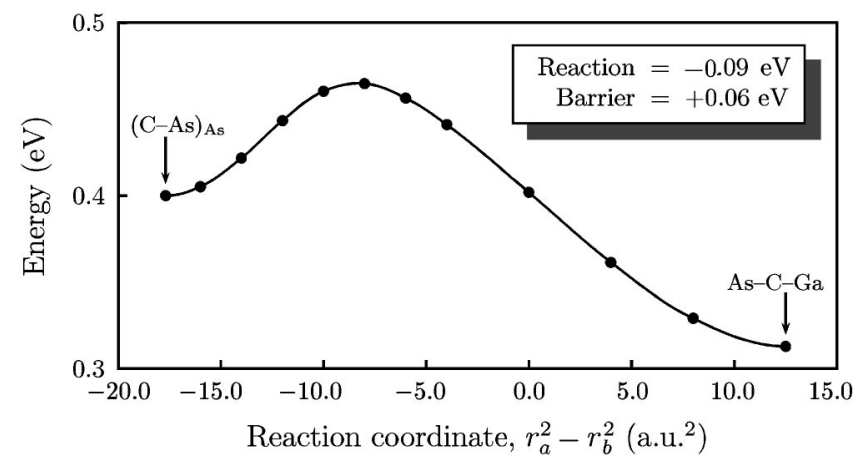

FIG. 3. Energy relative to the $(\mathrm{C}-\mathrm{Ga})_{\mathrm{Ga}}^{2+}$ complex plotted as a function of the reaction coordinate $r_{a}^{2}-r_{b}^{2}$ described in the main text and Fig. 2. The reaction energy and barrier height are relative to the initial $(\mathrm{C}-\mathrm{As})_{\mathrm{As}}^{2+}$ state.

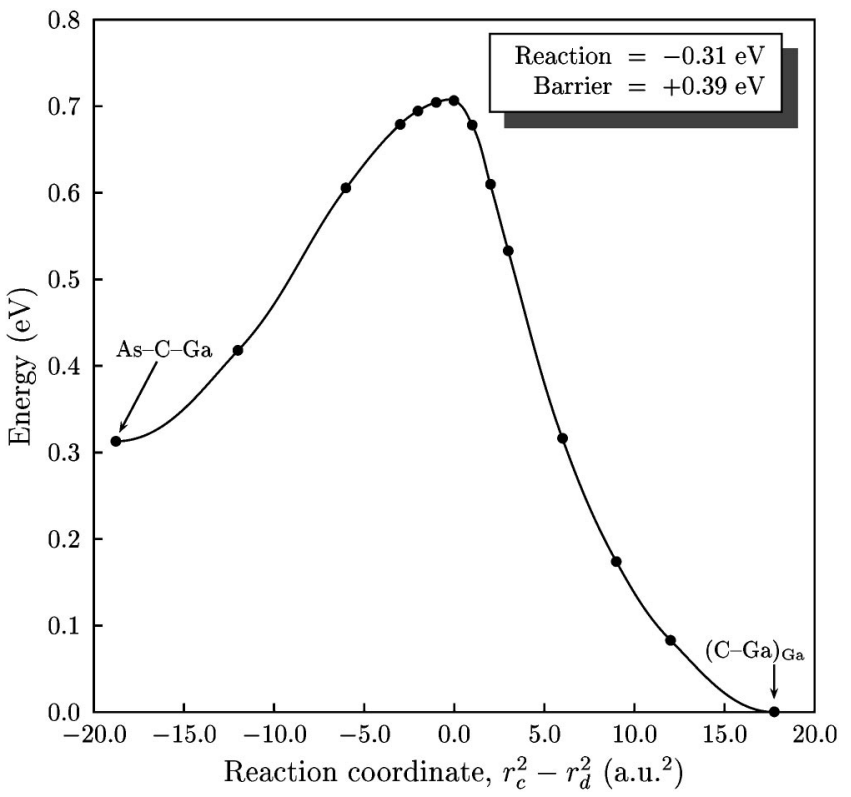

FIG. 4. Energy relative to the $(\mathrm{C}-\mathrm{Ga})_{\mathrm{Ga}}^{2+}$ complex plotted as a function of the reaction coordinate $r_{c}^{2}-r_{d}^{2}$ described in the main text and Fig. 2. The reaction energy and barrier height are relative to the intermediate $(\mathrm{Ga}-\mathrm{C}-\mathrm{As})^{2+}$ state.

\section{SUMMARY AND CONCLUSIONS}

Calculations using the SCC-DFTB method located three metastable minima in energy for a doubly-ionized intersitial carbon atom in GaAs. The three states; a $(\mathrm{C}-\mathrm{As})_{\mathrm{As}}^{2+}$ split interstitial, a bond-centered $(\mathrm{Ga}-\mathrm{C}-\mathrm{As})^{2+}$ interstitial, and a $(\mathrm{C}-\mathrm{Ga})_{\mathrm{Ga}}^{2+}$ split interstitial; were also found to be metastable using the AIMPRO method. Both methods found the same energy difference $(\approx 0.4 \mathrm{eV})$ between the two split interstitial defects to within $0.1 \mathrm{eV}$. The relative energy of the bondcentered interstitial compared with the split interstitials was a few tenths eV lower using the SCC-DFTB method than the AIMPRO method. It may be that the minimal basis approximation used by the SCC-DFTB method models this unusual linear structure less well than the split interstitial states resulting in a slight overbinding.

The SCC-DFTB method was then used to search for migration paths of carbon atoms in GaAs and identify the changes in bonding which occur during the process. It was found that starting from a $(\mathrm{C}-\mathrm{As})_{\mathrm{As}}^{2+}$ split interstitial state, a carbon atom moved first to the center of a $\mathrm{Ga}-\mathrm{As}$ bond, then into a $(\mathrm{C}-\mathrm{Ga})_{\mathrm{Ga}}^{2+}$ split interstitial state. These simulations showed two pairs of bonds were exchanged (i.e., broke and formed) during the migration process, with one pair in each half, and where the $(\mathrm{Ga}-\mathrm{C}-\mathrm{As})^{2+}$ interstitial is the intermediate state. The activation energies for each half of the reaction were then calculated using the AIMPRO method to be 0.09 and $0.39 \mathrm{eV}$, taking the differences of the squares of the lengths of the exchanged bonds to be the reation coordinates. Once a carbon atom reaches the $(\mathrm{C}-\mathrm{Ga})_{\mathrm{Ga}}^{2+}$ split interstitial state, it must then overcome a barrier of $0.71 \mathrm{eV}$ to return to a $(\mathrm{Ga}-\mathrm{C}-\mathrm{As})^{2+}$ interstitial state. This figure reperesents reasonable agreement with the value of $\approx 1 \mathrm{eV}$ measured by Fushimi and Wada for the hole loss process observed by them considering the uncertainties in the experiment and the approximations used by the theory. The simulations are also 
provide a mechanism for the formation of both $(\mathrm{C}-\mathrm{C})_{i}^{2+}$ and $(\mathrm{C}-\mathrm{C})_{\mathrm{As}}^{+}$dicarbon defects. Thus we conclude that once arsenic interstitials are present, the kick-out process originally proposed by H. M. You et al. initiates the diffusion of carbon atoms in GaAs via split interstitial and bond-centered interstitial states. The enhanced diffusion of carbon by this mechanism will only be sustained so long as the source of arsenic interstitials is not exhausted. We suggest a similar process also accounts for the formation of dicarbon defects in AlAs.

\section{ACKNOWLEDGMENT}

The Engineering and Physical Sciences Research Council (EPSRC), UK, is thanked for its financial support at Exeter University (Contract No. GR/L34457).
*Electronic address: C.D.Latham@ex.ac.uk

${ }^{1}$ B. T. Cunningham, L. J. Guido, J. E. Baker, J. S. Major, N. Holonyak, and G. E. Stillman, Appl. Phys. Lett. 55, 687 (1989).

${ }^{2}$ T. H. Chiu, J. E. Cunningham, J. A. Ditzenberger, W. Y. Jan, and S. N. G. Chu, J. Cryst. Growth 111, 274 (1991).

${ }^{3}$ H. M. You, T. Y. Tan, U. M. Gösele, S. T. Lee, G. E. Höfler, K. C. Hsieh, and N. Holonyak, J. Appl. Phys. 74, 2450 (1993).

${ }^{4}$ J. A. Zhou, C. Y. Song, J. F. Zheng, M. Stavola, C. R. Abernathy, and S. J. Pearton, Mater. Sci. Forum 258-263, 1293 (1997)

${ }^{5}$ S. P. Westwater and T. J. Bullough, J. Cryst. Growth 170, 752 (1997).

${ }^{6}$ G. E. Höfler, J. N. Baillargeon, K. C. Hsieh, and K. Y. Cheng, Appl. Phys. Lett. 60, 1990 (1992).

${ }^{7}$ G. E. Höfler and K. C. Hsieh, Appl. Phys. Lett. 61, 327 (1992).

${ }^{8}$ G. E. Höfler, H. J. Höfler, N. Holonyak, and K. C. Hsieh, J. Appl. Phys. 72, 5318 (1992).

${ }^{9}$ T. J. Delyon, J. M. Woodall, M. S. Goorsky, and P. D. Kirchner, Appl. Phys. Lett. 56, 1040 (1990).

${ }^{10}$ K. Watanabe and H. Yamazaki, Appl. Phys. Lett. 59, 434 (1991).

${ }^{11}$ J. D. Mackenzie, C. R. Abernathy, S. J. Pearton, and S. N. G. Chu, Appl. Phys. Lett. 66, 1397 (1995).

${ }^{12}$ D. L. Sato, F. J. Szalkowski, and H. P. Lee, Appl. Phys. Lett. 66, 1791 (1995).

${ }^{13}$ J. Wagner, R. C. Newman, B. R. Davidson, S. P. Westwater, T. J. Bullough, T. B. Joyce, C. D. Latham, R. Jones, and S. Öberg, Phys. Rev. Lett. 78, 74 (1997).

${ }^{14}$ C. D. Latham, R. Jones, B. R. Davidson, R. C. Newman, C. C. Button, P. R. Briddon, and S. Öberg, Phys. Status Solidi B 210, 869 (1998).

${ }^{15}$ B. R. Davidson, R. C. Newman, J. Wagner, C. D. Latham, R. Jones, C. C. Button, and P. R. Briddon, Phys. Rev. B 605447 (1999).

${ }^{16}$ H. Fushimi and K. Wada, J. Appl. Phys. 82, 1208 (1997).

${ }^{17}$ J. E. Northrup and S. B. Zhang, Phys. Rev. B 47, 6791 (1993).

${ }^{18}$ H. Fushimi and K. Wada, IEEE Trans. Electron Devices 44, 1996 (1997)

${ }^{19}$ S. J. Breuer, R. Jones, P. R. Briddon, and S. Öberg, Phys. Rev. B 53, 16289 (1996).

${ }^{20}$ Y. Cheng, M. Stavola, C. R. Abernathy, S. J. Pearton, and W. S. Hobson, Phys. Rev. B 49, 2469 (1994).

${ }^{21}$ B. Clerjaud, F. Gendron, M. Krause, and W. Ulrici, Phys. Rev.
Lett. 65, 1800 (1990)

${ }^{22}$ R. Jones and S. Öberg, Phys. Rev. B 44, 3673 (1991).

${ }^{23}$ U. Gösele, T. Y. Tan, M. Schultz, U. Egger, P. Werner, R. Scholz, and O. Breitenstein, Defect Diffus. Forum 143, 1079 (1997).

${ }^{24}$ D. Porezag, T. Frauenheim, T. Köhler, G. Seifert, and R. Kaschner, Phys. Rev. B 51, 12947 (1995).

${ }^{25}$ M. Elstner, D. Porezag, G. Jungnickel, J. Elsner, M. Haugk, Th. Frauenheim, S. Shuhai, and G. Seifert, Phys. Rev. B 58, 7260 (1998).

${ }^{26}$ J. Elsner, R. Jones, P. K. Sitch, V. D. Porezag, M. Elstner, T. Frauenheim, M. I. Heggie, S. Öberg, and P. R. Briddon, Phys. Rev. Lett. 79, 3672 (1997).

${ }^{27}$ G. B. Bachelet, D. R. Hamann, and M. Schlüter, Phys. Rev. B 26, 4199 (1982).

${ }^{28}$ M. I. Heggie, C. D. Latham, S. C. P. Maynard, and R. Jones, Chem. Phys. Lett. 249, 485 (1996).

${ }^{29} \mathrm{R}$. Jones and P. R. Briddon, in Identification of Defects in Semiconductors, Vol. 51A of Semiconductors and Semimetals, edited by M. Stavola (Academic Press, Boston, 1998), Chap. 6.

${ }^{30}$ G. D. Watkins and K. L. Brower, Phys. Rev. Lett. 36, 1329 (1976).

${ }^{31}$ L. W. Song and G. D. Watkins, Phys. Rev. B 42, 5759 (1990).

${ }^{32}$ A. K. Tipping and R. C. Newman, Semicond. Sci. Technol. 2, 315 (1987).

${ }^{33}$ P. Leary, R. Jones, S. Öberg, and V. J. B. Torres, Phys. Rev. B 55, 2188 (1997).

${ }^{34}$ J. Tersoff, Phys. Rev. Lett. 64, 1757 (1990).

${ }^{35}$ R. B. Capaz, A. Dalpino, and J. D. Joannopoulos, Phys. Rev. B 50, 7439 (1994).

${ }^{36}$ A. Mainwood, Mater. Sci. Forum 196-201, 1589 (1995).

${ }^{37}$ P. A. Stolk, H.-J. Grossman, D. J. Eaglesham, D. C. Jacobson, C. S. Rafferty, G. H. Gilmer, M. Jaraíz, and J. M. Poate, J. Appl. Phys. 81, 6031 (1997).

${ }^{38}$ J. D. Collins, G. A. Gledhill, R. Murray, P. S. Nandhra, and R. C. Newman, Phys. Status Solidi B 151, 469 (1989).

${ }^{39}$ G. A. Gledhill, S. B. Upadhyay, M. J. L. Sangster, and R. C. Newman, J. Mol. Struct. 247, 313 (1991).

${ }^{40}$ R. B. Beall, R. C. Newman, J. E. Whitehouse, and J. Woodhead, J. Phys. C 17, 2653 (1984).

${ }^{41}$ R. Jones and S. Öberg, Semicond. Sci. Technol. 7, 855 (1992). 